\title{
SOBRE A FORÇA DE ÁCIDOS E BASES: ALGUMAS CONSIDERAÇÕES
}

\author{
Eduardo J. S. Vichi ${ }^{\dagger}$ e Aécio Pereira Chagas* \\ Instituto de Química, Universidade Estadual de Campinas, CP 6154, 13084-971 Campinas - SP, Brasil
}

Recebido em 20/11/07; aceito em 31/1/08; publicado na web em 13/8/08

\begin{abstract}
ON THE STRENGTH OF ACIDS AND BASES: SOME CONSIDERATIONS. This article intends to answer the question: "what is the best way to evaluate the strength of acids and bases?" The meaning of the word strength, the main acid-base theories (ionotropic and electron pair), the neutralization reactions and the thermodynamical formalism are considered. Some cases are presented and discussed. In conclusion, evaluating acid-base strength is dependent on the theory (formalism) as well as on the system and measuring techniques.
\end{abstract}

Keywords: acid-base strength; acid-base theory; chemical formalism.

\section{INTRODUÇÃO}

O tema ácido-base é, sem dúvida, um dos mais importantes da Química. Como consequiência, muitas são as teorias e os debates envolvendo fatos antigos e novos e sempre há o que se destacar, haja visto dois livros que devem ser mencionados: o de Leach ${ }^{1}$ e o de Costa et al.. ${ }^{2}$ Muitas vezes participamos de discussões envolvendo colegas e estudantes, nas salas, nos corredores e em outros locais menos formais e um tema sempre ressurgente é a força de ácidos e bases. Daí a razão deste artigo.

No caso, a pergunta central é: "qual a melhor maneira de se medir a força de um ácido ou de uma base?" Vamos tentar discutir um pouco essa questão e, depois, mostrar também a importância dos formalismos químicos. Entretanto, antes de entrarmos no assunto principal, são indispensáveis alguns preliminares.

\section{Preliminares}

Inicialmente é necessário explicar o que se entende por força. Este termo inicialmente tem uma origem antropomórfica, metamorfoseando-se depois, nos séculos XVIII e XIX, com mais de um significado dentro das ciências, sem mencionar na linguagem comum. No presente caso, apesar da mesma origem, o termo força não tem nada a ver com a mesma palavra utilizada em Mecânica, com sentido diverso. Considera-se que a força de um ácido ou de uma base é a intensidade ou o valor (maior ou menor) de uma propriedade característica dos mesmos, conforme será visto. O uso do termo força também subentende a utilização dos atributos forte e fraco. A polissemia não é privilégio da linguagem comum.

Outro ponto a ser lembrado são as características do comportamento ácido-base, brilhantemente sintetizadas por Lewis em 1938: ${ }^{3} \mathrm{a}$ reação entre um ácido e uma base (neutralização) é rápida; um ácido (ou uma base) pode deslocar um ácido (ou uma base) mais fraco de seus compostos; ácidos e bases podem ser titulados um com o outro por meio de indicadores; ácidos e bases são capazes de atuarem como catalisadores.

As atuais teorias ácido-base, utilizadas para explicar este comportamento, já incorporam estes critérios em seus conceitos e estrutura, bem como aspectos constitucionais e/ou moleculares.

\footnotetext{
+ In memoriam

* e-mail: aecio@iqm.unicamp.br
}

\section{AS TEORIAS ÁCIDO-BASE E SEUS FORMALISMOS}

Para facilitar nossa discussão, pode-se dizer que há, atualmente, duas teorias principais para explicar o comportamento ácido-base das substâncias, ${ }^{4,5}$ resumido por Lewis e acima citado. A estrutura de todas as teorias é semelhante: comporta uma definição de ácido e de base, que apresentam uma simetria inversa, uma definição de reação de neutralização (a reação tipo entre um ácido e uma base) e, evidentemente, uma heurística e uma casuística ${ }^{6}$ próprias da aplicação destes conceitos aos fatos já conhecidos e por conhecer. Entretanto, não considerando estes aspectos heurísticos e casuísticos, algumas teorias podem ser reduzidas a outras, nos seus aspectos formais e é isto que será enfatizado nesta discussão. As duas teorias principais são a teoria ionotrópica e a teoria do par eletrônico. As outras teorias podem ser consideradas como casos particulares destas, principalmente da primeira o que, o ponto de vista dos autores, não irá alterar estas considerações. Cada uma dessas teorias tem seu formalismo, como será visto, e este é fundamental para a medida da força ácido-base.

A teoria ionotrópica é uma generalização das teorias de Brønsted (próton), Lux (óxido) e outras. A reação de neutralização (vide critérios de Lewis) pode ser representada pela Equação:

$\mathrm{A}_{1}+\mathrm{B}_{2} \leftrightarrows \mathrm{B}_{1}+\mathrm{A}_{2}$

em que $A_{1}$ e $B_{1}$ são as formas ácida e básica do sistema 1 e $A_{2}$ e $B_{2}$ respectivamente do sistema 2 . Pode ser ainda considerada como a soma de duas outras equações:

$$
\begin{aligned}
\mathrm{A}_{1} & \rightleftarrows \mathrm{B}_{1}+\theta \\
\mathrm{B}_{2}+\theta & \rightleftarrows \mathrm{A}_{2}
\end{aligned}
$$

$\mathrm{A}_{1}+\mathrm{B}_{2} \rightleftarrows \mathrm{B}_{1}+\mathrm{A}_{2}$

A partícula transferida $\theta$ pode ser $\mathrm{o} \mathrm{H}^{+}$ou outro cátion. Quando a partícula $\theta$ for negativa. como $\mathrm{O}^{2-}, \mathrm{Cl}^{-}$etc., tem-se que inverter o que é considerado ácido e base, ou seja, base é a doadora de $\theta$.

Na teoria do par eletrônico ou de Lewis, a reação de neutralização pode ser representada pela Equação:

$\mathrm{A}+: \mathrm{B} \leftrightarrows \mathrm{A}: \mathrm{B}$ 
sendo A o ácido, :B a base e : representa um par de elétrons.

Na teoria ionotrópica, a reação de neutralização é uma reação de dupla troca. A partícula $\theta$ vai do sistema 1 para o sistema 2 . A espécie que "com ela está" é a base. Por exemplo:

$$
\mathrm{HAc}+\mathrm{H}_{2} \mathrm{O} \rightleftarrows \mathrm{Ac}^{-}+\mathrm{H}_{3} \mathrm{O}^{+}
$$

$$
\mathrm{A}_{1}+\mathrm{B}_{2} \rightleftarrows \mathrm{B}_{1}+\mathrm{A}_{2}
$$

em que $\mathrm{Ac}^{-}$é o ânion acetato, sendo transferida a partícula $\mathrm{H}^{+}$(o próton, segundo Brønsted). Aqui a base mais forte é aquela que retém a partícula. No sistema real, formado por um grande número de partículas, haverá uma distribuição estatística do $\mathrm{H}^{+}$entre $\mathrm{H}_{2} \mathrm{O}$ e $\mathrm{Ac}^{-}$, sendo que a base mais forte (no caso $\mathrm{Ac}^{-}$) reterá a maior fração de $\mathrm{H}^{+}$que a outra $\left(\mathrm{H}_{2} \mathrm{O}\right)$, mais fraca.

$\mathrm{Na}$ teoria do par eletrônico, a neutralização é uma reação de síntese, o par eletrônico da base é doado ao ácido, formando então um aducto ou complexo (este nome varia conforme o contexto), em que o par eletrônico é compartilhado. Por exemplo:

$\mathrm{BCl}_{3}(\mathrm{~g})+: \mathrm{NH}_{3}(\mathrm{~g}) \leftrightarrows \mathrm{Cl}_{3} \mathrm{~B}: \mathrm{NH}_{3}(\mathrm{~g})$

Considerando um determinado ácido como referência, uma base 1 será mais forte que uma base 2 quanto mais forte (ver abaixo o significado deste termo) for a ligação formada no complexo e analogamente, quando se comparam dois ou mais ácidos, utilizando sempre uma mesma base como referência.

\section{Um pouco de Termodinâmica}

As variações das funções termodinâmicas energia livre, $G$, entalpia, $H$ e entropia, $S$, em um dado sistema (ver símbolos no final do artigo), podem ser interpretadas qualitativamente de um ponto de vista molecular. Estas funções estão relacionadas pela conhecida equação:

$$
\mathrm{G}=\mathrm{H}-\mathrm{TS} \text { ou } \Delta_{\mathrm{r}} \mathrm{G}=\Delta_{\mathrm{r}} \mathrm{H}-\mathrm{T} \Delta_{\mathrm{r}} \mathrm{S} \quad(\mathrm{T} \text { e p constantes) }
$$

As variações da função $H$, sempre a $T$ e $p$ constantes, podem ser associadas às interações atômicas e moleculares no sistema, como a formação e quebra de ligações químicas. As variações da função $S$ estão associadas à distribuição de energia: quanto mais níveis energéticos disponíveis há no sistema, maior será a entropia e, portanto, em transformações em que há aumento de entropia, há um aumento dos níveis disponíveis para a energia se distribuir e vice-versa. As variações da função $G$ refletem um compromisso entre estes dois tipos de transformações, como se pode notar na Equação 6. Uma transformação termodinamicamente favorável, ou seja transformação em que $\Delta G<0$, dependerá dos valores e sinais das outras duas funções $H$ e $S$ (além de $T$ ). Uma discussão um pouco mais detalhada sobre o assunto o leitor poderá encontrar na ref. 7.

Na reação de neutralização da teoria ionotrópica (dupla troca), há a quebra de uma ligação entre $A_{1}$ e $\theta$ e a formação da ligação entre $\theta$ e $B_{2}$. Se as bases $B_{1}$ e $B_{2}$ têm afinidades próximas pela partícula $\theta$, então a diferença de entalpia associada à ligação rompida e a formada será pequena, ou seja $\Delta_{\mathrm{r}} H \cong 0$, por mais forte que sejam as ligações envolvidas, pois a quebra de uma (processo endotérmico) é compensada pela formação da outra (processo exotérmico). Se as afinidades forem muito diferentes, $\Delta_{r} H$ já será apreciável. Entretanto, a distribuição estatística de $\theta$ entre as duas bases será mais facilmente detectável e, portanto, a variação de $\Delta S$ será mais interessante que a de $\Delta_{r} H$. Como medidas de $\Delta_{\mathrm{r}} G$ são mais factíveis que de $\Delta_{\mathrm{r}} S$ e estes termos estão relacionados pela Equação 6 (e $\Delta_{\mathrm{r}} H \cong 0$ ), a primeira função é então utilizada na grande maioria das vezes. Obviamente qualquer outra grandeza diretamente proporcional a $\Delta_{\mathrm{r}} G$ pode ser utilizada, como a constante de equilíbrio (ou seu logaritmo) associada ao sistema, a posição de equilíbrio de um sistema indicador, a atividade de uma ou mais espécies (como o logarítmo da atividade do $\mathrm{H}_{3} \mathrm{O}^{+}$, ou $\mathrm{pH}$ ), constantes cinéticas (a lei de Brønsted da catálise ácido-base), ${ }^{8}$ força eletromotriz de uma célula eletroquímica etc.

Em resumo, na teoria ionotrópica, a posição de equilíbrio correspondente à reação de neutralização é a maneira mais conveniente de medir a força relativa de bases em relação a um ácido ou vice-versa.

A neutralização, segundo a teoria do par eletrônico, é uma reação de síntese, onde há a formação de uma nova ligação. $\Delta_{r} G$ será quase que exclusivamente dependente de $\Delta_{\mathrm{r}} H$, pois o termo $T \Delta_{\mathrm{r}} S$ será praticamente constante na maior parte das reações (em fase gasosa corresponderia à perda de três graus de liberdade, $3 / 2 R T$ ). Então a força ácido-base será proporcional à estabilidade da ligação formada e esta poderá ser medida por $\Delta_{\mathrm{r}} \mathrm{H}$ ou outro parâmetro que possa caracterizar uma ligação química, como distância interatômica, densidade eletrônica etc., ou ainda grandezas proporcionais a estas, estabelecidas empiricamente, como, por exemplo, o deslocamento de uma banda espectral. De modo geral, três grandezas são as mais utilizadas para caracterizar uma ligação química entre dois átomos $\mathrm{M}$ e N: a energia associada à formação da ligação M-N (ou a dissociação dos mesmos), a distância interatômica M-N e a distribuição da densidade eletrônica entre $\mathrm{Me} \mathrm{N}$ e, em princípio, qualquer parâmetro que possa ser tomado como uma medida de uma dessas grandezas pode também ser utilizado para medir a força ácido-base de Lewis. Em resumo, na teoria do par eletrônico a maneira mais conveniente de medir a força relativa de uma base com outra em relação a um ácido é caracterizando a ligação formada, ou analogamente para comparar dois ou mais ácidos.

\section{O CASO DA ÁGUA}

Consideremos novamente a Equação 1 e vamos aplicar a lei dos equilíbrios químicos ou lei da ação das massas ao sistema:

$K=a\left(\mathrm{~B}_{1}\right) a\left(\mathrm{~A}_{2}\right) / a\left(\mathrm{~A}_{1}\right) a\left(\mathrm{~B}_{2}\right)$

Em um sistema aquoso em que $\mathrm{B}_{2}=\mathrm{H}_{2} \mathrm{O}, a\left(\mathrm{~B}_{2}\right) \cong 1$ (soluções diluidas) e $a\left(\mathrm{~A}_{2}\right)=a\left(\mathrm{H}_{3} \mathrm{O}^{+}\right)$, a Equação 7 torna-se:

$K=a\left(\mathrm{~B}_{1}\right) a\left(\mathrm{H}_{3} \mathrm{O}^{+}\right) / a\left(\mathrm{~A}_{1}\right)$

sendo que a utilização e a medida de $a\left(\mathrm{H}_{3} \mathrm{O}^{+}\right)$são bastante convenientes. A Equação 8 pode ser escrita na forma logarítmica:

$\log K=\log \left[a\left(\mathrm{~B}_{1}\right) / a\left(\mathrm{~A}_{1}\right)\right]+\log a\left(\mathrm{H}_{3} \mathrm{O}^{+}\right)$

utilizando a notação $-\log x=p x$, esta Equação torna-se:

$\mathrm{pH}=\mathrm{p} K+\log \left[a\left(\mathrm{~A}_{1}\right) / a\left(\mathrm{~B}_{1}\right)\right]$

fórmula bastante conhecida e utilizada em sistemas aquosos.

Então, a medida da força ácido-base depende da teoria utilizada, ou seja, do formalismo da reação de neutralização e da interpretação molecular das variações das funções termodinâmicas.

Há ainda a considerar as circunstâncias do sistema em estudo, como as fases presentes, a possibilidade de se utilizar uma determinada técnica ou não etc. Como exemplos, a medida do $\mathrm{pH}$ em sistemas aquosos, a utilização da Equação de Langmuir em sistemas com uma interface sólido-gás ou sólido-líquido, ${ }^{9}$ a determinação da afinidade protônica, ${ }^{10}$ ou seja: 
$\mathrm{H}^{+}(\mathrm{g})+: \mathrm{B}(\mathrm{g}) \leftrightarrows \mathrm{H}^{\mathrm{B}} \mathrm{B}^{+}(\mathrm{g}) ; \Delta \mathrm{H}^{\theta} \equiv$ afinidade protônica

utilizando técnicas para estudo de interações íon-molécula (espectrometria de massa e congêneres). A afinidade protônica é também considerada uma medida absoluta da basicidade de um substância.

Como se sabe, a teoria do par eletrônico é mais abrangente que a ionotrópica, porém, em sistemas que podem ser tratados por uma teoria ou outra, a escolha é muitas vezes determinada pelas circunstâncias acima mencionadas.

\section{UMA ANOMALIA}

A escolha e a utilização destes formalismos, para se comparar ácidos e bases, está condicionada às restrições de cada um: $\Delta H \cong 0$ para o formalismo ionotrópico e $\Delta_{\mathrm{r}} S$ constante para o formalismo do par eletrônico, além da única reação envolvida ser a neutralização. Quando estas condições não são satisfeitas surgem as anomalias.

Airoldi e Santos, ${ }^{11}$ estudando a interação entre algumas bases orgânicas de Lewis e sílica gel, com seu grupo silanol ( $\equiv \mathrm{Si}-\mathrm{OH})$ comportando-se com ácido, em solução de 1,2 dicloroetano (DCE), ou seja:

$\equiv \mathrm{Si}-\mathrm{OH}(\mathrm{s})+: \mathrm{B}(\mathrm{sln}) \leftrightarrows \equiv \mathrm{Si}-\mathrm{OH}: \mathrm{B}(\mathrm{s})+$ solv

encontraram os resultados que estão na Tabela 1 . Nesta estão apresentados os valores de $D N$ (Donor Number, de Gutmann) ${ }^{12}$ e os valores de $\Delta_{r} H$, $\Delta_{\mathrm{r}} G \mathrm{e} \Delta_{\mathrm{r}} S$ correspondentes à reação representada pela Equação 12. Como se pode observar, a menos do metanol, os valores de $D N$ e de $-\Delta_{\mathrm{r}} H$ crescem na mesma direção. Os valores de $\Delta S$ não são regulares, mas afetam menos $\Delta_{r} G$ que o termo entálpico. O metanol apresenta um valor de $-\Delta_{r} H$ bem mais alto que as outras bases, há uma grande diminuição do valor de $\Delta S$ e, entretanto, enquadra-se na regularidade de $\Delta_{r} G$. Airoldi e Santos atribuem a anomalia do metanol a uma provável formação de clusters de hidrogênio ligados. Há também que se considerar o efeito do solvente, que nem sempre é pouco solvatante, ${ }^{13,14}$ a heterogeneidade da superfície e o uso do modelo de Langmuir. ${ }^{9}$

Tabela 1. Parâmetros termodinâmicos da interação de sílica-gel com algumas bases orgânicas em 1,2-dicloroetano a $298 \mathrm{~K}$ e $0,1 \mathrm{MPa}$ (baseada na ref. 11)

\begin{tabular}{lcccc}
\hline Base & $D N^{*}$ & $\begin{array}{c}-\Delta_{\mathrm{r}} \mathrm{H} / \\
\mathrm{kJ} \mathrm{mol}^{-1}\end{array}$ & $\begin{array}{c}-\Delta_{\mathrm{r}} \mathrm{G} / \\
\mathrm{kJ} \mathrm{mol}^{-1}\end{array}$ & $\begin{array}{c}\Delta \mathrm{S} / \\
\mathrm{J} \mathrm{K}^{-1} \mathrm{~mol}^{-1}\end{array}$ \\
\hline Acetonitrila & 14,1 & 1,28 & 2,24 & 4,03 \\
Metanol & 19,0 & 12,78 & 2,40 & $-34,56$ \\
Acetona & 17,0 & 1,29 & 2,69 & 3,98 \\
N,N-dimetilformamida & 26,6 & 2,25 & 2,89 & 0,77 \\
N,N-dimetilacetamida & 27,8 & 5,84 & 3,07 & $-11,26$ \\
Piridina & 33,1 & 7,09 & 3,07 & $-15,50$ \\
\hline * Donor Number (ver ref.12) & & &
\end{tabular}

\section{Estendendo o formalismo}

Mencionou-se que na teoria ionotrópica a partícula transferida $\theta$ poderia ser: $\mathrm{H}^{+}, \mathrm{O}^{2-}, \mathrm{Cl}^{-}$(Equação 2 ). A estas pode-se acrescentar também o elétron, $\mathrm{e}^{-}$, este mesmo formalismo e algumas equações termodinâmicas bem conhecidas, para serem utilizados nas reações de óxido-redução. Exemplificando:

$$
\begin{gathered}
\mathrm{Fe}^{2+} \rightleftarrows \mathrm{Fe}^{3+}+\mathrm{e}^{-} \\
\mathrm{Ag}^{+}+\mathrm{e}^{-} \rightleftarrows \mathrm{Ag} \\
\mathrm{Fe}^{2+}+\mathrm{Ag}^{+} \rightleftarrows \mathrm{Ag}+\mathrm{Fe}^{3+}
\end{gathered}
$$

aplicando a lei de ação das massas:

$K=a\left(\mathrm{Fe}^{3+}\right) a(\mathrm{Ag}) / a\left(\mathrm{Fe}^{2+}\right) a\left(\mathrm{Ag}^{+}\right)$

escrevendo na forma logarítmica:

In $K=\ln \left[a\left(\mathrm{Fe}^{3+}\right) / a\left(\mathrm{Fe}^{2+}\right)\right]+\ln \left[a(\mathrm{Ag}) / a\left(\mathrm{Ag}^{+}\right)\right]$

Multiplicando-se ambos os membros dessa equação por $-R T / v\left(\mathrm{e}^{-}\right) F E$, em que $R=$ constante dos gases, $T=$ temperatura termodinâmica, $v\left(\mathrm{e}^{-}\right)=$coeficiente estequiométrico do elétron, $F=$ constante de Faraday e $E=$ força eletromotriz da célula eletroquímica associada à reação, tem-se:

$\left[R T / v\left(\mathrm{e}^{-}\right) F E\right] \ln K=\left[R T / \mathrm{v}\left(\mathrm{e}^{-}\right) F E\right] \ln \left[a\left(\mathrm{Fe}^{3+}\right) / a\left(\mathrm{Fe}^{2+}\right)\right]+\left[R T / \mathrm{v}\left(\mathrm{e}^{-}\right)\right.$ $F E] \ln \left[a(\mathrm{Ag}) / a\left(\mathrm{Ag}^{+}\right)\right]$

lembrando que

$\Delta_{\mathrm{r}} G^{\theta}=-R T \ln K^{\theta}$ (“Equação isoterma de Van't Hoff”) ${ }^{7,15}$

e

$\Delta \mathrm{r} G^{\theta}=-v\left(\mathrm{e}^{-}\right) F E^{\theta}$ ("Equação fundamental da Eletroquímica") ${ }^{7,15}$

substituindo-se estas duas últimas equações na Equação 16 e simplificando, tem-se

$E^{\theta}=E\left(\mathrm{Fe}^{3+} / \mathrm{Fe}^{2+}\right)+E\left(\mathrm{Ag} / \mathrm{Ag}^{+}\right)$

A Equação 19, que permite calcular a força eletromotriz de uma pilha, é usualmente obtida através da Equação de Nernst e as respectivas definições de "potencial de eletrodo" associadas.?

Este formalismo pode ser estendido também para outras situações. Convidamos o leitor a considerar a teoria ácido-base de Usanovich, bem como a de Ingold. ${ }^{5}$

\section{CONCLUSÃO}

Finalizando a presente discussão, pode-se concluir que a medida da força de um ácido ou de uma base depende da teoria (reação de neutralização) que se está considerando, de outras circunstâncias relativas ao próprio sistema e às técnicas de medida.

Notar a "força heurística" destes formalismos inerentes às teorias, porém é preciso não esquecer que o mesmo é termodinâmico, macroscópico, e não representa um mecanismo no nível molecular. Esta confusão é muito comum e, muitas vezes, leva a equívocos e ambigüidades, dificultando a aprendizagem e utilização destas teorias e conceitos. Assim também, a interpretação molecular das funções termodinâmicas, aqui considerada, é qualitativa e pequenas alterações não implicam necessariamente em alterações maiores nos aspectos quantitativos das medidas e das equações.

O objetivo da presente discussão foi tentar aclarar a questão da força de ácidos e bases e os conceitos envolvidos e não propriamente conduzir a uma visão puramente formalista do problema e da Química em geral. Como foi mencionado, cada teoria tem sua heurística e casuística próprias e este formalismo não pretende quebrá-las.

\section{AGRADECIMENTOS}

Os autores agradecem ao Prof. C. Airoldi pela discussão e valiosas sugestões. 


\section{SÍMBOLOS UTILIZADOS}

$\Delta_{\mathrm{r}} X \equiv \sum_{\mathrm{B}} \mathrm{v}_{\mathrm{B}} X$, sendo $v_{\mathrm{B}}$ o coeficiente estequiométrico da espécie $\mathrm{B}$, associado à equação química genérica $0=\sum_{\mathrm{B}} \mathrm{V}_{\mathrm{B}} \mathrm{B}$.

$X=$ funções termodinâmicas de estado: $G, H, S, S T$.

$G$ = função de Gibbs ou energia livre de Gibbs.

$H=$ entalpia.

$S=$ entropia.

$T=$ temperatura termodinâmica.

$p=$ pressão.

$p^{\theta}=$ pressão padrão $=0,1 \mathrm{MPa}$. O expoente ${ }^{\theta}$ em outras funções $\left(G^{\theta}\right.$, $H^{\theta}$ ) denota-as também como padrão (para mais detalhes ver ref. 7).

$K=$ constante de equilíbrio termodinâmica.

$E=$ força eletromotriz de uma célula galvânica.

$F=$ constante de Faraday.

$R=$ constante dos gases.

\section{REFERÊNCIAS E NOTAS}

1. Leach, M. R.; Lewis Acid/Base Reaction Chemistry, Meta-Synthesis. Com: Brighton (U.K.), 1999.

2. Costa, P. R. R.; Ferreira, V. F.; Esteves, P. M.; Vasconcellos, M. L. A.; Ácidos e Bases em Química Orgânica, Bookman: Porto Alegre, 2005.

3. Lewis, G. L.; J. Franklin Inst. 1938, 226, 293
4. Chagas, A. P.; Quim. Nova 2000, 23, 126

5. Jensen, W. B.; The Lewis Acid-Base Concepts, J. Wiley \& Sons: New York, 1980.

6. Estamos entendendo estes vocábulos como: heurística é o conjunto de regras e métodos que conduzem à descoberta, à invenção e à resolução de problemas e casuística é a discussão e/ou análise dos casos dentro de um determinado contexto que podem levar à ampliação da compreensão deste contexto e/ou esclarecimento de outros casos (ver ref. 4).

7. Chagas, A. P.; Termodinâmica Química, Editora da Unicamp: Campinas, 1999.

8. Dill, K. A.; Bromberg, S.; Molecular Driving Forces, Garland Science: New York, 2003.

9. Chagas, A. P.; Simoni, J. de A. Em Encyclopedia of Surface \& Colloid Science; Hubbard, A., ed.; Marcel Dekker: New York, 2002, p. 54555364.

10. Drago, R. S.; Matwiyoff, N. A.; Acidos y Bases, Editorial Reverté: México, 1972.

11. Airoldi, C.; Santos Jr., L. S.; Termochim. Acta 1986, 104, 111.

12. Gutmann, V.; The Donor-Acceptor Approach to Molecular Interactions, Plenum Press: New York, 1978.

13. Olofsson, G.; Olofsson, I.; J. Am. Chem. Soc. 1973, 95, 7231.

14. Rolim, G. L.; Simoni, J. A.; Chagas, A. P.; J. Braz. Chem. Soc. 1990, 1, 95.

15. Simoni, J. de A.; Chagas, A. P.; Quim. Nova 2007, 30, 501. 